\title{
25 Research Square \\ Early liver injury is closely related to organ failure of sepsis
}

\section{Milin Peng}

Xiangya Hospital Central South University https://orcid.org/0000-0002-5443-5646

\section{Yuhang Ai}

Xiangya Hospital Central South University

\section{Lina Zhang}

Xiangya Hospital Central South University

Shuangping Zhao

Xiangya Hospital Central South University

Zhiyong Liu

Xiangya Hospital Central South University

Haisong Zhang

Xiangya Hospital Central South University

Xiaolei Zhang

Xiangya Hospital Central South University

Desheng Qi ( $\sim$ qidesheng17@csu.edu.cn )

\section{Research article}

Keywords: Sepsis, Early liver injury, Organ failure, Risk factor

Posted Date: January 24th, 2020

DOI: https://doi.org/10.21203/rs.2.21859/v1

License: (c) (i) This work is licensed under a Creative Commons Attribution 4.0 International License.

Read Full License 


\section{Abstract}

Backgrounds: Sepsis induced organ failure is main cause of mortality in intensive care units (ICU), however, the impact of early liver injury on clinical ending of sepsis is not clear and has not been discussed in context of clinical research on sepsis before. Our study aimed at the investigation of the clinical effect of early liver injury within $48 \mathrm{~h}$ at ICU admission on sepsis outcomes.

Methods: A single-centered, retrospective cohort of 198 adult critical patients diagnosed with sepsis were included in different ICU departments of Xiangya hospital from 2016 to 2018. Patients were divided into two groups, early liver injury and non-early liver injury within $48 \mathrm{~h}$ at ICU admission. Baseline characteristics, clinical outcomes and risk factors of these two groups were studied. Logistic regression analysis, Cox hazard analysis, Kaplan-Meier and log-rank test were used.

Results: In total, 198 patients with sepsis were included, with 106 (53.5\%) with early liver injury and overall in-hospital mortality was $45.9 \%(n=91)$. Compared to non-early liver injury group, patients with early liver injury had significant higher SOFA (7.44 \pm 3.83 vs. $5.55 \pm 2.61, P<0.001)$, APACHE II score (15.22 \pm 23.14 vs. $9.14 \pm 8.72, P=0.013)$, procalcitonin ( $37.10 \pm 59.20$ vs. $19.24 \pm 48.10, P=0.021)$, and rate of shock $(63.2 \%$ vs. $48.9 \%, P=0.046)$. Primary outcome showed that early liver injury group had significant higher rate of renal dysfunction ( 62.3 vs. $33.7 \%, P<0.001)$, coagulation $(31.1 \%$ vs. $13.0 \%$, $\mathrm{P}=0.004)$ and hematologic system $(72.6 \%$ vs. $52.2 \%, \mathrm{P}=0.003)$ dysfunction hinting at higher organ failure rate. Age (OR 0.966; 95\% Cl 0.944-0.989; $P=0.004$ ), hypertriglyceridemia (OR 1.636; 95\% Cl 1.049-2.551; $\mathrm{P}=0.030$ ), serum total bile acid (TBA, OR 1.071; $95 \% \mathrm{Cl}$ 1.030-1.113; $\mathrm{P}=0.001)$, hemoglobin (OR 1.030; $95 \% \mathrm{Cl} 1.013-1.046 ; \mathrm{P}<0.001$ ) and renal dysfunction (OR 3.403; 95\% $\mathrm{Cl} 1.631-7.099 ; \mathrm{P}=0.001$ ) were the independent predictors for early liver injury in sepsis by multiple regression analysis. Kaplan-Meier survival analyze demonstrated early liver injury and non-early liver injury group had similar survival time.

Conclusion: Early liver injury within 48 hours on ICU admission is closely related to organ failure for patients with sepsis. Future study with big data is required to verify our viewpoint.

\section{Background}

Sepsis is a life-threatening organ dysfunction caused by a dysregulated host response to infection (1), which has the high incidence and mortality rate among diseases in ICU. According to the recent worldwide epidemiological study, sepsis incidence rate was about $39 \%$ in ICU. Overall ICU and hospital mortality rate of sepsis were $25.8 \%$ and $35.3 \%$, respectively, varied among different continents (2). Although the certain effect of clinical therapy, morbidity and mortality rates in sepsis remain high, triggering us to investigate deeper understanding of sepsis pathogenesis and novel treatment.

Liver plays a central role in sepsis as it is essential for clearing bacteria and toxins,mediating inflammatory responses, and coagulating (3). Yet its protective response also causes liver injury, and liver injury often occurs in early stage of sepsis because of inflammation and hypoperfusion. Liver injury will lead to the decrease of bacteria and toxins clearance, exacerbating the severity of sepsis $(3,4)$. Patients 
with pre-existing liver disease or liver failure, like cirrhosis, trauma, and drug-induced liver injury, are associated with higher risks of sepsis morbidity, multiple organ failure (MOF) and sepsis-induced mortality than those without liver diseases (5-9). Attenuating liver injury maybe beneficial to lower sepsis mortality rates by regulating immune responses for clearing bacterias and helping other organs withstand cascading injury.

Yet to date, the impact of early liver injury on clinical outcomes in sepsis remains unknown. Considering the underestimated pivotal role of early liver injury in the development of sepsis, the objective of this study is to determine the effect of early hepatic injury on sepsis outcomes.

\section{Methods}

\section{Study Design and Population}

Study participants were at different ICU departments of Xiangya Hospital Central South University of China between January 2016 to February 2018. The setting of this study were different ICU departments including General ICU, Respiratory ICU, Emergency ICU, Neurosurgical ICU and other ICUs. The Institutional Review Board of Xiangya Hospital Central South University approved the study. The study was conducted in accordance with STrengthening the Reporting of OBservational studies in Epidemiology (STROBE) statement. The inclusion criteria were patients with sepsis, 18 years or older, and at hospital admission more than $48 \mathrm{~h}$. Exclusion criteria was patients with pre-existing severe liver dysfunction, chronic liver diseases, such as liver cirrhosis, viral hepatitis.

\section{Data source}

Data of patients were retrospectively collected from Electronic Hospital Records System (EHRs) with clinical data, including age, gender, pre-existing disease, source of infection; laboratory biochemical parameters within $48 \mathrm{~h}$ on admission with blood routine, liver and kidney function, coagulation function, procalcitonin, etc; sequential organ failure assessment (SOFA) score, and Acute Physiology, Age, Chronic Health Evaluation II (APACHE II) score; rates of organ failure; complications with septic shock, acute respiration distress syndrome (ARDS), acute kidney injury (AKI); the use of continuous renal replacement therapy (CRRT), length of vasopressor use; length of hospital stays, and mortality at hospital. Follow up with 180-day and 1-year mortality was prospectively collected after discharge. Patients were grouped according to the liver function at $48 \mathrm{~h}$ admission.

\section{Variable definitions}

The definition of sepsis was life-threatening organ dysfunction caused by a dysregulated host response to infection (SOFA score of $\geq 2$ ) and septic shock was defined as a combination of hypotension requiring vasopressor support to maintain a MAP of $65 \mathrm{mmHg}$ and a lactate greater than $2 \mathrm{mmol} / \mathrm{L}$ despite fluid resuscitation, according to Sepsis 3.0 criteria (10). Early liver injury was diagnosed in patients with new onset of plasma $\square$ serum bilirubin $>2 \mathrm{mg} / \mathrm{dL}$, or, Alanine transaminase (ALT) or Aspartate transaminase $($ AST $)>$ twice the normal value $(80 \mathrm{U} / \mathrm{L})$ within $48 \mathrm{~h}$ of admission. Organ failure was defined as organ 
exhaustion except liver. Hypertriglyceridemia was defined as the level of serum triglyceride with above the upper limit of normal. Acute respiration distress syndrome (ARDS) was defined by Berlin criteria of 2012 (11), and AKI diagnosis criteria adopted KDIGO clinical practice guideline: increase in serum creatinine of $\geq 0.3 \mathrm{mg} / \mathrm{dL}$ or $\geq 50 \%$ within 48 hours or urine output of $<0.5 \mathrm{~mL} / \mathrm{kg} /$ hour for $>6$ hours (12). According to SOFA score system, the definition of hematologic dysfunction was diagnosed with the level of platelet $<150 * 10^{\wedge} 9 / \mathrm{L}$, respiration dysfunction with $\mathrm{p} 02 / \mathrm{FiO} 2 \leq 400$ or needing mechanism ventilation, neurological dysfunction with Glasgow Coma Score (GCS) $<14$, cardiovascular dysfunction with mean arterial pressure (MAP) $<70 \mathrm{mmHg}$ or needing vasoactive drug, renal dysfunction with creatinine $\geq$ $1.2 \mathrm{mg} / \mathrm{dL}$ (13). Coagulation dysfunction was defined with prothrombin time above 3 times the upper limit of normal or activated partial thromboplastin time (APTT) prolonger $10 \mathrm{~s}$ above the upper limit of normal value.

\section{Outcomes}

Our primary outcome was organ failure. Secondary outcomes were all-cause mortality, complications of ARDS, AKI and septic shock; the use of CRRT, the use of vasopressor, length of vasopressor use, length of hospital stays, the use of mechanical ventilation (MV), MV time and length of hospital days.

\section{Statistical analysis}

Statistical analysis was performed using SPSS 23 (SPSS, Inc., Chicago, IL). All description data are presented as median (Standard deviation) or frequencies (\%). Student's t-test or Wilcoxon's rank-sum test if appropriate was used to compare quantitative variables between groups. The chi-square test was used for categorical variables. Kaplan-Meier survival analysis was performed according to early liver injury or not. The predictive values of early liver injury of sepsis were assessed by univariate and multiplevariate logistic regression models and to identify the independent variables affecting early liver injury. The predictors for mortality in sepsis were assessed by univariate and stepwise multivariate Cox proportional hazards moels. Cox regression model was used to adjust for residual imbalance by including parameters with $p<0.05$ and potential confounders judged by clinical expertise. P value of $<0.05$ (two-sided) was considered significant.

\section{Results}

We identified 198 individuals with sepsis who met the inclusion criteria, during a 48-month period from 2016 to 2018. In this cohort, 91 patients died at hospital discharge (45.9\%), 107 patients died at 180 days, and 113 patients died at 1 year (68.4\%) after hospital discharge. Of the 198 patients, early liver injury within $48 \mathrm{~h}$ admission, had occurred in 106 (53.5\%) patients. Characteristics of clinical and biological data are presented in Table 1. 
Table 1

Baseline characteristics of early liver injury group and control group (non-early liver injury) within $48 \mathrm{~h}$ on admission of sepsis patients

\begin{tabular}{|c|c|c|c|}
\hline & $\begin{array}{l}\text { Early liver injury } \\
\text { group } \\
(n=106)\end{array}$ & $\begin{array}{l}\text { Control group } \\
(n=92)\end{array}$ & $\mathbf{P}$ \\
\hline \multicolumn{4}{|l|}{ Baseline information } \\
\hline Age, median (SD) & $55.72(15.72)$ & $62.82(15.71)$ & 0.002 \\
\hline Male, n (\%) & $69(65.1)$ & $48(52.2)$ & 0.082 \\
\hline Respiratory rate, median (SD) & $24.87(7.16)$ & $22.80(5.87)$ & 0.028 \\
\hline Heart rate, median (SD) & 111.66(101.46) & $111.92(129.36)$ & 0.990 \\
\hline GCS, median (SD) & $11.97(4.17)$ & $12.26(3.79)$ & 0.784 \\
\hline $\begin{array}{l}\text { Pre-exiting conditions } \\
\text { Connective tissue disease }\end{array}$ & $5(4.7)$ & $3(3.3)$ & 0.727 \\
\hline Gastrointestinal hemorrhage & $5(4.7)$ & $2(2.2)$ & 0.453 \\
\hline Coronary heart disease & 12(11.3) & $17(18.5)$ & 0.165 \\
\hline Hypertension & $28(26.4)$ & $26(28.3)$ & 0.873 \\
\hline \multicolumn{4}{|l|}{ Infection source, $\mathrm{n}(\%)$} \\
\hline Urinary system & $22(21.8)$ & 14(15.6) & 0.354 \\
\hline Abdomen & $8(7.5)$ & $14(15.2)$ & 0.113 \\
\hline $\begin{array}{l}\text { Respiratory tract } \\
\text { Critical Score }\end{array}$ & $58(54.7)$ & $58(63.0)$ & 0.250 \\
\hline SOFA score, median (SD) & $7.44(3.83)$ & $5.55(2.61)$ & $\begin{array}{l}< \\
0.001\end{array}$ \\
\hline APACHE II score, median (SD) & $15.22(23.14)$ & $9.14(8.72)$ & 0.013 \\
\hline
\end{tabular}

Continuous variables are reported as mean.

Abbreviations: SOFA Sequential Organ Failure Assessment, APACH II Acute Physiology and Chronic Health Evaluation II, WBC White Blood Cell, N(\%) neutrophil percentage, ALT alanine transaminase, AST aspartate transaminase, ALB albumin, TB total bilirubin, DB direct bilirubin, GGT gammaglutamyl transpeptidase, AKP alkline phosphatase, TBA total bile acid, TG triglyceride, $\mathrm{CHOL}$ cholesterol, LDL low density lipoprotein, HDL high density lipoprotein, CK creatine kinase, CK-MB creatine kinase-MB, LDH lactate dehydrogenase, Mb myoglobin, NTproBNP N-terminal of the prohormone brain natriuretic peptide, APTT activated partial thromboplastin time, PT prothrombin time, PTA prothrombin time activity percentage, GCS Glasgow Coma Scale. 


$\begin{array}{ll}\begin{array}{l}\text { Early liver injury } \\ \text { group }\end{array} & \begin{array}{l}\text { Control group } \\ (n=92)\end{array} \\ (n=106) & \end{array}$

Blood routine examination, median (SD)

\begin{tabular}{|c|c|c|c|}
\hline WBC $\left(10^{\wedge} 9 / \mathrm{L}\right)$ & 14.04(10.96) & $14.27(13.62)$ & 0.894 \\
\hline $\mathrm{N}(\%)$ & $81.69(16.48)$ & 81.94(13.79) & 0.909 \\
\hline Platelet (10^9/L) & 111.92(95.04) & 168.03(108.75) & $\begin{array}{l}< \\
0.001\end{array}$ \\
\hline Hemoglobin & $103.40(24.64)$ & $95.37(22.57)$ & 0.019 \\
\hline Lymphocyte count & $0.95(1.20)$ & $1.19(0.76)$ & 0.895 \\
\hline Monocyte count & $0.90(1.49)$ & $0.66(0.63)$ & 0.127 \\
\hline \multicolumn{4}{|c|}{ Liver function, median (SD) } \\
\hline Serum ALT & 253.22(659.37) & $22.60(13.65)$ & $\begin{array}{l}< \\
0.001\end{array}$ \\
\hline Serum AST & $390.19(1026.21)$ & $35.82(18.34)$ & 0.001 \\
\hline Serum ALB & 29.83(21.35) & $29.02(5.92)$ & 0.723 \\
\hline Serum TBIL & $54.53(80.37)$ & $13.39(7.87)$ & $\begin{array}{l}< \\
0.001\end{array}$ \\
\hline Serum DBIL & $29.43(44.54)$ & $7.48(11.76)$ & $\begin{array}{l}<.001 \\
0.001\end{array}$ \\
\hline Serum TBA & 19.58(33.14) & $6.82(7.25)$ & $\begin{array}{l}<.001 \\
0.001\end{array}$ \\
\hline Serum GGT & $177.55(178.54)$ & $47.61(51.26)$ & 0.001 \\
\hline Serum AKP & $278.72(178.55)$ & $138.22(160.24)$ & 0.113 \\
\hline \multicolumn{4}{|c|}{ Blood lipid examination, median (SD) } \\
\hline Serum TG & $2.01(3.13)$ & $1.12(1.03)$ & 0.007 \\
\hline
\end{tabular}

Continuous variables are reported as mean.

Abbreviations: SOFA Sequential Organ Failure Assessment, APACH II Acute Physiology and Chronic Health Evaluation II, WBC White Blood Cell, N(\%) neutrophil percentage, ALT alanine transaminase, AST aspartate transaminase, ALB albumin, TB total bilirubin, DB direct bilirubin, GGT gammaglutamyl transpeptidase, AKP alkline phosphatase, TBA total bile acid, TG triglyceride, $\mathrm{CHOL}$ cholesterol, LDL low density lipoprotein, HDL high density lipoprotein, CK creatine kinase, CK-MB creatine kinase-MB, LDH lactate dehydrogenase, Mb myoglobin, NTproBNP N-terminal of the prohormone brain natriuretic peptide, APTT activated partial thromboplastin time, PT prothrombin time, PTA prothrombin time activity percentage, GCS Glasgow Coma Scale. 


\begin{tabular}{|c|c|c|c|}
\hline & $\begin{array}{l}\text { Early liver injury } \\
\text { group } \\
(n=106)\end{array}$ & $\begin{array}{l}\text { Control group } \\
(n=92)\end{array}$ & $\mathbf{P}$ \\
\hline Serum $\mathrm{CHOL}$ & $2.37(2.19)$ & $2.53(1.89)$ & 0.572 \\
\hline Serum LDL & $2.71(14.11)$ & $1.65(1.72)$ & 0.476 \\
\hline Serum HDL & $0.46(0.47)$ & $1.66(8.20)$ & 0.136 \\
\hline Serum creatinine, median (SD) & 204.58(166.12) & $150.62(179.39)$ & 0.029 \\
\hline \multicolumn{4}{|l|}{ Myocardial enzymology, median (SD) } \\
\hline CK & 1036.83(3129.68) & $1120.59(245.88)$ & 0.003 \\
\hline CK-MB & $52.43(86.84)$ & $26.15(71.52)$ & 0.021 \\
\hline LDH & 1030.72(2266.89) & $359.61(290.65)$ & 0.003 \\
\hline $\mathrm{Mb}$ & $310.44(759.98)$ & 135.94(193.05) & 0.034 \\
\hline NT-proBNP & 6136.63(17485.35) & $5831.24(15229.21)$ & 0.914 \\
\hline \multicolumn{4}{|l|}{ Coagulation examination, median (SD) } \\
\hline APTT & 43.38(18.93) & $37.96(8.91)$ & 0.011 \\
\hline PT & $16.68(4.80)$ & $15.31(3.03)$ & 0.016 \\
\hline PTA & $80.28(66.43)$ & 100.93(139.19) & 0.176 \\
\hline $\begin{array}{l}\text { Infection and inflammatory indicators, median } \\
\text { (SD) } \\
\text { Procalcitonin, median (SD) }\end{array}$ & $37.10(59.20)$ & 19.24(48.10) & 0.021 \\
\hline $\begin{array}{l}\text { C-reaction protein, median (SD) } \\
\text { Lactate, median (SD) }\end{array}$ & $\begin{array}{l}85.84(114.66) \\
2.43(2.91)\end{array}$ & $\begin{array}{l}97.32(175.96) \\
1.51(1.74)\end{array}$ & $\begin{array}{l}0.584 \\
0.007\end{array}$ \\
\hline \multicolumn{4}{|l|}{ Continuous variables are reported as mean. } \\
\hline \multicolumn{4}{|c|}{$\begin{array}{l}\text { Abbreviations: SOFA Sequential Organ Failure Assessment, APACH II Acute Physiology and Chronic } \\
\text { Health Evaluation II, WBC White Blood Cell, N(\%) neutrophil percentage, ALT alanine transaminase, } \\
\text { AST aspartate transaminase, ALB albumin, TB total bilirubin, DB direct bilirubin, GGT gamma- } \\
\text { glutamyl transpeptidase, AKP alkline phosphatase, TBA total bile acid, TG triglyceride, CHOL } \\
\text { cholesterol, LDL low density lipoprotein, HDL high density lipoprotein, CK creatine kinase, CK-MB } \\
\text { creatine kinase-MB, LDH lactate dehydrogenase, Mb myoglobin, NTproBNP N-terminal of the } \\
\text { prohormone brain natriuretic peptide, APTT activated partial thromboplastin time, PT prothrombin } \\
\text { time, PTA prothrombin time activity percentage, GCS Glasgow Coma Scale. }\end{array}$} \\
\hline
\end{tabular}

\section{Baseline characteristics of early liver injury group and control group (non-early liver injury) within $48 \mathrm{~h}$ on}




\section{admission of septic patients}

We found that younger septic patients were more prone to early liver damage ( $55.72 \pm 15.72$ vs. $62.82 \pm$ $15.71, P=0.002$ ). There was no significant difference in pre-existing diseases and infection source between early liver injury and non-early liver injury groups. Septic patients with early liver injury showed significant higher severity of illness, with higher SOFA score $(7.44 \pm 3.83$ vs. $5.55 \pm 2.61, P<0.001)$ and APACHE II score (15.22 \pm 23.14 vs. $9.14 \pm 8.72, \mathrm{P}=0.013)$, higher infectious indicators (Procalcitonin, $37.10 \pm 59.20$ vs. $19.24 \pm 48.10, P=0.021$ ), when compared to non-early liver injury group. Furthermore, patients with early liver injury had a significantly higher levels of lactate $(2.43 \pm 2.91$ vs. $1.51 \pm 1.74, \mathrm{P}=$ 0.007 ), total bile acid (TBA, $19.58 \pm 33.14$ vs. $6.82 \pm 7.25, P<0.001$ ), triglyceride (TG, $2.01 \pm 3.13$ vs. 1.12 $\pm 1.03, P=0.007)$, creatinine (204.58 \pm 166.12 vs. $150.62 \pm 179.39, P=0.029)$, APTT activated partial thromboplastin time $(43.38 \pm 18.93$ vs. $37.96 \pm 8.91, \mathrm{P}=0.011)$, PT prothrombin time $(16.68 \pm 4.80$ vs. $15.31 \pm 3.03, P=0.016)$, and lower levels of platelet $(111.92 \pm 95.04$ vs. $168.03 \pm 108.75, P<0.001)$.

\section{Effect of early liver injury on clinical outcomes and complications in sepsis}

The primary outcome, as shown in Table 2, demonstrated that there were significant higher rates of organ failure in patients with early liver injury group, with the occurrence of renal dysfunction ( $62.3 \mathrm{vs}$. $33.7 \%, P$ $<0.001)$, hematologic dysfunction $(72.6 \%$ vs. $52.2 \%, P=0.003)$, and coagulation dysfunction $(31.1 \%$ vs. $13.0 \%, P=0.004)$ compared to non-early liver injury group. 
Table 2

Effect of early liver injury on clinical outcomes and complications in sepsis

$\begin{array}{ll}\text { Early liver injury( }(n=106) & \begin{array}{l}\text { Non-early liver } \\ \text { injury } \\ (n=92)\end{array}\end{array}$

Primary outcome

Organ failure, $\mathrm{n}(\%)$

Neurologic dysfunction

$12(41.4)$

11(37.9)

1.000

Cardiovascular dysfunction

$67(63.2)$

$46(50.0)$

0.064

Hematologic dysfunction

$77(72.6)$

48(52.2)

0.003

Renal dysfunction

66(62.3)

31(33.7)

$<$

0.001

Coagulation dysfunction

33(31.1)

12(13.0)

0.004

Respiration dysfunction

65(61.3)

63(68.5)

0.302

Secondary outcomes

Complications, n (\%)

\begin{tabular}{llll}
\hline ARDS & $35(33.0)$ & $21(22.8)$ & 0.117 \\
\hline AKI & $56(52.8)$ & $26(28.3)$ & 0.001 \\
\hline Septic shock & $67(63.2)$ & $45(48.9)$ & 0.046 \\
\hline Hypertriglyceridemia, $\mathrm{n}(\%)$ & $38(35.8)$ & $20(21.7)$ & 0.010 \\
\hline CRRT, n (\%) & $31(29.2)$ & $15(16.3)$ & 0.042 \\
\hline MV, n (\%) & $60(56.6)$ & $44(47.8)$ & 0.254 \\
\hline MV time (h), median (SD) & $101.29(236.32)$ & $95.39(162.11)$ & 0.841 \\
\hline Length of hospital stays(d), median (SD) & $8.46(12.01)$ & $10.37(21.11)$ & 0.461 \\
\hline Vasopressor drug use, $\mathrm{n}(\%)$ & $58(54.7)$ & $40(43.5)$ & 0.120 \\
\hline Length of vasopressor drug (d), median & $2.98(4.76)$ & $1.52(2.456)$ & 0.006 \\
\hline SD) & & & \\
\hline Mortality, $\mathrm{n}(\%)$ & & $38(41.3)$ & 0.140 \\
\hline At hospital & $53(50.0)$ & & \\
\hline
\end{tabular}

Categorical variables are reported as count (\% of column total)

Abbreviations: CRRT Continuous renal replacement therapy, ARDS Acute respiratory distress syndrome, AKI Acute kidney injury, MV time Mechanical ventilation time. 


\begin{tabular}{|c|c|c|c|}
\hline & Early liver injury $(n=106)$ & $\begin{array}{l}\text { Non-early liver } \\
\text { injury } \\
(n=92)\end{array}$ & $\mathbf{P}$ \\
\hline 180 days & $61(67.8)$ & $46(61.3)$ & 0.563 \\
\hline 1 year & $63(70.0)$ & $50(66.7)$ & 0.735 \\
\hline \multicolumn{4}{|c|}{ Categorical variables are reported as count (\% of column total) } \\
\hline
\end{tabular}

In the secondary outcomes, early liver injury group had higher rates of complications with septic shock ( $63.2 \%$ vs. $48.9 \%, P=0.046)$, AKI ( $52.8 \%$ vs. $28.3 \%, P=0.001)$; higher rates of hypertriglyceridemia $(35.8 \%$ vs. $21.7 \%, P=0.010)$, prolonger length of vasopressor use $(2.98 \pm 4.76 \mathrm{~d}$ vs. $1.52 \pm 2.456 \mathrm{~d}, P=0.006)$ and the use of CRRT ( $29.2 \%$ vs. $16.3 \%, P=0.042$, Table 2$)$ compared to non-early liver injury group. Non-early liver injury and early liver injury group had similar mortality, with hospital mortality $50.0 \%$ in early liver injury group, and $41.3 \%$ in non-early liver injury group $(P=0.140), 180$ days mortality after discharge $67.8 \%$ in early liver injury group, and $61.3 \%$ in nog-early liver injury group $(P=0.563)$, one year mortality after discharge $70.0 \%$ in early liver injury group, and $66.7 \%$ in the control group $(P=0.735)$. Furthermore, Kaplan-Meier survival estimate was performed to analyze survival rate difference between early liver injury group and non-early liver injury group. There was no significant difference between these two groups within total survival time of 1 year (Fig. 1. $\left.X^{2}=1.338, P>0.05\right)$.

\section{Univariate and multiple regression analysis to explore risk factors affecting early liver injury in sepsis}

In the univariate logistic regression analysis, age, septic shock, SOFA score, APACHE II score, serum procalcitonin, TBA, lactate, platelet, and hemoglobin, hypertriglyceridemia, renal dysfunction were significant predictors of early liver injury in sepsis. Further in the multivariate stepwise logistic regression analysis, age was the only independent protect predictors for early liver injury in sepsis (OR 0.966; $95 \% \mathrm{Cl}$ $0.944-0.989 ; \mathrm{P}=0.004$, Table 3$)$. The results also showed that hypertriglyceridemia (OR $1.636 ; 95 \% \mathrm{Cl}$ $1.049-2.551 ; \mathrm{P}=0.030$ ), the occurrence of renal dysfunction (OR 3.403; 95\% Cl 1.631-7.099; $\mathrm{P}=0.001$ ), hemoglobin (OR 1.030; 95\% Cl 1.013-1.046; $\mathrm{P}<0.001)$ and serum TBA levels (OR 1.071; $95 \% \mathrm{Cl} 1.030-$ $1.113 ; P=0.001$ ) were the independent risk factors impacting on new onset of early liver injury within $48 \mathrm{~h}$ admission in sepsis, indicating hypertriglyceridemia and cholestasis were closely associated with liver injury in early stage of sepsis. 
Table 3

Univariate and multivariate regression analysis to explore predictors of early liver injury in sepsis

\begin{tabular}{|c|c|c|c|c|c|c|}
\hline & \multicolumn{2}{|c|}{ Univariate analysis } & \multicolumn{3}{|c|}{ Multiple analysis } & \multirow[b]{2}{*}{$\mathrm{P}$} \\
\hline & OR & $95 \% \mathrm{Cl} \quad \mathrm{P}$ & OR & & $95 \% \mathrm{Cl}$ & \\
\hline Age (year) & 0.972 & $0.954-0.990$ & 0.002 & 0.966 & $0.944-0.989$ & 0.004 \\
\hline Septic shock & 1.794 & $1.016-3.167$ & 0.044 & - & & \\
\hline SOFA & 1.189 & $1.084-1.304$ & $<0.001$ & - & & \\
\hline APACHE II & 1.026 & $1.002-1.049$ & 0.031 & 1.027 & $0.992-1.063$ & 0.128 \\
\hline \multicolumn{7}{|l|}{ Laboratory parameters } \\
\hline Serum PCT & 1.007 & $1.001-1.013$ & 0.028 & - & & \\
\hline Serum total bile acid & 1.060 & $1.023-1.099$ & 0.001 & 1.071 & $1.030-1.113$ & 0.001 \\
\hline lactate & 1.196 & $1.037-1.379$ & 0.014 & 1.213 & $1.008-1.459$ & 0.041 \\
\hline Serum hemoglobin & 1.015 & $1.002-1.027$ & 0.020 & 1.030 & $1.013-1.046$ & $<0.001$ \\
\hline Hypertriglyceridemia & 0.641 & $0.451-0.912$ & 0.013 & 1.636 & $1.049-2.551$ & 0.030 \\
\hline \multicolumn{7}{|l|}{ Organ failure } \\
\hline Coagulation dysfunction & 3.014 & $1.448-6.272$ & 0.003 & - & & \\
\hline Hematologic dysfunction & 2.434 & $1.347-4.397$ & 0.003 & - & & \\
\hline Renal dysfunction & 3.247 & $1.810-5.822$ & $<0.001$ & 3.403 & $1.631-7.099$ & 0.001 \\
\hline
\end{tabular}

\section{Univariate and multivariate Cox hazard analysis to explore risk factors of mortality in sepsis}

In the multivariate Cox hazard analysis (Table 4), age (HR 1.014; 95\% Cl 1.000-1.028; $\mathrm{P}=0.044$ ), organ failure of cardiovascular dysfunction (HR 1.673;95\% $\mathrm{Cl} 1.065-2.629 ; \mathrm{P}=0.026)$, respiration dysfunction (HR 2.435; 95\% Cl 1.455-4.073; $\mathrm{P}=0.001)$ were independent risk factors for one year mortality of septic patients after discharge, while SOFA, APACHE II, lactate, hypertriglyceridemia, early liver injury, septic shock renal, hemotological and coagulation dysfunction were not. 
Table 4

Univariate and multivariate Cox hazard analysis to explore risk factors of mortality in sepsis

\begin{tabular}{|c|c|c|c|c|c|c|}
\hline & \multicolumn{2}{|c|}{ Univariate analysis } & \multicolumn{3}{|c|}{ Multiple analysis } & \multirow[b]{2}{*}{$\mathrm{P}$} \\
\hline & HR & $95 \% \mathrm{Cl} \quad \mathrm{P}$ & HR & & $95 \% \mathrm{Cl}$ & \\
\hline Age (year) & 1.015 & $1.002-1.028$ & 0.024 & 1.014 & $1.000-1.028$ & 0.044 \\
\hline SOFA & 1.110 & $1.057-1.167$ & $<0.001$ & - & & \\
\hline APACHE II & 1.008 & $1.001-1.015$ & 0.032 & 1.027 & $0.992-1.063$ & 0.128 \\
\hline \multicolumn{7}{|l|}{ Laboratory parameters } \\
\hline Serum PCT & 0.998 & $0.994-1.001$ & 0.998 & - & & \\
\hline Serum total bile acid & 1.000 & $0.991-1.008$ & 0.947 & - & & \\
\hline lactate & 1.101 & $1.025-1.182$ & 0.008 & - & & \\
\hline Serum hemoglobin & 0.988 & $0.980-0.997$ & 0.007 & - & & \\
\hline Hypertriglyceridemia & 1.255 & $1.006-1.566$ & 0.044 & - & & \\
\hline Multi-Organ dysfunction & 4.504 & $2.273-8.925$ & $<0.001$ & - & & \\
\hline Early liver injury & 1.112 & $0.768-1.610$ & 0.573 & - & & \\
\hline Cardiovascular dysfunction & 2.515 & $1.661-3.806$ & $<0.001$ & 1.673 & $1.065-2.629$ & 0.026 \\
\hline Coagulation dysfunction & 1.598 & $1.047-2.439$ & 0.030 & - & & \\
\hline Respiration dysfunction & 3.382 & $2.097-5.455$ & $<0.001$ & 2.435 & $1.455-4.073$ & 0.001 \\
\hline Hematologic dysfunction & 1.594 & $1.070-2.374$ & 0.022 & - & & \\
\hline Renal dysfunction & 1.308 & $0.905-1.891$ & 0.153 & - & & \\
\hline Neurologic dysfunction & 2.714 & $1.402-5.254$ & 0.003 & - & & \\
\hline \multicolumn{7}{|l|}{ Complications } \\
\hline AKI & 1.147 & $0.791-1.663$ & 0.470 & - & & \\
\hline Septic shock & 2.429 & $1.611-3.662$ & $<0.001$ & - & & \\
\hline ARDS & 2.647 & $1.800-3.892$ & $<0.001$ & 1.483 & $0.978-2.249$ & 0.064 \\
\hline Vasoactive drug usage & 2.467 & $1.668-3.647$ & $<0.001$ & - & & \\
\hline CRRT & 2.438 & $1.630-3.647$ & $<0.001$ & - & & \\
\hline $\begin{array}{l}\text { Abbreviations: SOFA, sequen } \\
\text { Health Evaluation II, PCT, Pro } \\
\text { injury. }\end{array}$ & $\begin{array}{l}\text { ial organ } \\
\text { calcitonir }\end{array}$ & $\begin{array}{l}\text { failure assessm } \\
\text { ARDS, Acute re }\end{array}$ & $\begin{array}{l}\text { APACHE } \\
\text { ratory dist }\end{array}$ & $\begin{array}{l}\text { I, Acut } \\
\text { ess syl }\end{array}$ & $\begin{array}{l}\text { hysiology, Age, } \\
\text { ome, AKI Acute }\end{array}$ & $\begin{array}{l}\text { hronic } \\
\text { idney }\end{array}$ \\
\hline
\end{tabular}




\section{Discussion}

In this retrospective cohort study, first we found the onset of early hepatic injury within 48 hours on ICU admission was closely related to organ failure for patients with sepsis. Secondly, age, hypertriglyceridemia, serum total bile acid, hemoglobin and renal dysfunction were the independent predictors for early liver injury in sepsis by multiple regression analysis, while Kaplan-Meier survival analysis demonstrated similar survival time between early liver injury and non-early liver injury group. Third, age, organ failure of cardiovascular dysfunction, respiration dysfunction were independent risk factors for one year mortality of septic patients after discharge in the multivariate Cox hazard analysis.

The definition of liver injury of ours was different from traditional conception using bilirubin which can be explained as following. We demonstrated the percentage of septic patients with early hepatic injury within $48 \mathrm{~h}$ of admission was about $53.5 \%$, which is not consistent with $11 \%$ occurrence showed in other study (14). Unlike using bilirubin as the only marker to define early liver injury, we choosed bilirubin > $2 \mathrm{mg} / \mathrm{dL}$, or ALT or AST > twice the normal value representing early liver injury. Liver has powerful compensatory ability so early hepatic injury is latent. As bilirubin rises several days after hepatic dysfunction, we choose more sensitive indicators ALT or AST to define early liver injury. ALT was also identified as an independent predictive factor of critical illnesses (15). Moreover, hypoxia hepatitis, mainly histopathological changes in liver injury in sepsis (3), is characterized by marked increased ALT after insufficient oxygen uptake by hepatocytes (16). So we used the more sensitive indicators, ALT and AST, to present early and latent hepatic injury at the beginning of sepsis as liver has been ignored in sepsis compared to other organs. Our data have shown high incidence of $53.5 \%$ of early hepatic injury in septic patients. So, more attention should be paid to the liver, the guardian, modifier and target of sepsis (3).

Interestingly, we found younger septic patients were more prone to early liver damage in multivariate regression analysis (Table 3) which is at odds with results of Kramer's study (14). One of causes is that Kramer research included all ICU patients while we choose only septic patients. A multiplecenter study from Japan demonstrated average age of septic patients is $69 \pm 17$ years (6). The underlying factor is that Japan is a population aging society than our country, and different regions, races and genetic background are important factors influencing sepsis epidemiology results, while our data is from Chinese Han population. Recently a cross continents epidemiology research of sepsis reported that average age of septic patients is $61.5 \pm 17$ years (2). Liver is a critical site to modify the immune response in sepsis. And younger patients may have a stronger immune response against infection attack than older ones. So younger patients are possible more prone to early liver injury in sepsis and this argument needs further indepth research. While age was independent risk factor for mortality of septic patients after discharge for one year in multivariate Cox hazard analysis (Table 4), which was in line with previous detailed summary (17).

Our study showed ALT, AST, total bilirubin (TB), direct bilirubin (DB), gamma-glutamyl transpeptidase (GGT), TBA, triglyceride and lactate rise in septic patients with early liver injury (Table $1,3,4)$. Due to the great heterogeneity in clinical patients, the liver of individual has different tolerance ability to sepsis. Our 
clinical results hint at hepatitis, bile capillary obstruction, cholestasis, bile acid and lipid metabolism abnormity in septic patients with early liver injury. Sepsis influences both hepatic parenchymal and interstitial tissues and biliary tract. These results are consistent with the pathological change of liver in sepsis (18), 60\% patients had "hepatitis" type including portal/lobular inflammation, hemorrhagic and necrosis, $40 \%$ patients had "mixed" liver injury by combinations of biliary lesions, like cholangitis, and portal/lobular inflammation, $73.3 \%$ patients had steatosis which was robust evidence and golden criteria. Further, we put forward hypoxia hepatitis, cholestasis, bile acid and lipid metabolism abnormity are main pathological and metabolic characteristics of early liver injury of sepsis.

There are few researches studied the correlation between early liver injury and severity of sepsis before. To our data, early liver injury is closely related to severity of sepsis as higher SOFA and APACHE II score, infectious indicators like PCT, prolonging length of vasopressor days, higher rates of CRRT and septic shock, some of which are typical indicators for exacerbation of sepsis $(2,19,20)$. What' more, few clinical studies have focused on the role of liver in organ failure in sepsis. We find, compared to non-early liver injury group, early liver injury group has widely worsening renal, coagulation and hemotological dyfunction judged by details from SOFA criteria. Liver and kidney interaction, namedly liver-kidney axis or hepatorenal syndrome are often referred in severe hepatic disease or other diseases situation $(21,22)$, but rarely has been discussed in sepsis. Our results prompt the underlying close interaction between liver and kidney at the beginning of sepsis, which may stimulate related further studies in the future. Though hemotological system is often ignored in sepsis, platelet, one member of blood system, can not be overlooked in sepsis. To our data, early liver injury group has significant lower platelet. Platelet plays key role in initial step to blood clotting and maintaining homeostasis among coagulation, vascular endothelium and cytokine network (23). Platelet is also found to have important functions like immune surveillance, co-working with immune cells to fight against invading pathogens (24). And thrombocytopenia is closely associated with dysregulated host response and increased 30 day mortality in recent research (25). Significant decreasing platelet in early liver injury group in our results suggest that early hepatic injury can break hemotological homeostasis in early phase of sepsis. Bleeding and coagulation disorder plays a crucial part in progress of sepsis and can lead to disseminated intravascular coagulation (DIC) in late stage of sepsis. To our data, worsening coagulation function in early liver injury group hints at turbulent coagulation function may come up in early stage of sepsis, which is reasonable due to close innate relationship between liver and coagulation function. Albeit not significantly, there were tendency of dampened cardiovascular and respiratory function in early liver injury group showned by faster respiratory rate and higher myocardial enzyme level (Table 1), which would be latent due to infectious fever and hyperdynamic circulation at the beginning of sepsis. Serum hemoglobin level shown as independent risk factor for early liver injury of sepsis (Table 3 ) is in line with previous study on nonalcoholic liver disease (26), indicating the mutual effect of hemoglobin and liver and requiring further study.

Our results are in line with some recent basic studies. Liver plays a central role in bacterias clearance, coagulating, modifying inflammatory and immune response, mediating a large number of the metabolism process in sepsis. These critical biological functions have important impact on other organs 
and systems including kidney, lung, cardiovascular and coagulation systems, acid-base balance and lipid metabolism (27). And Liver regulates inflammatory response to infection, generating plentiful inflammatory cytokines to spread to other vital organs by blood circulation to adapt to "inflammatory need" to help other organs to fight against pathogens. Liver is also a school to "re-education" circulating immune cells staying in hepatic sinusoid temporarily that thereafter recycle between different organs to fulfill demands to withstand attack from pathogens invaders (28). In some cases, this important adaptive function of liver can loss control and overreact thus producing redundant inflammatory cytokines to damage other organs.

However, there are no significant difference in hospital mortality, 180 days or 1 year after discharge between early liver injury group and non-early liver injury group shown by our results, which are not consistent with other studies $(14,29)$. Due to the two studies design is different from us, one is including all critically ill patients (14), the other is discussing the impact of liver trauma on sepsis. Early hepatic injury was closely related to organ failure but not mortality shown by our results (Table 2,4 and Fig. 1). We suppose liver is the first sentry and processor to face exogenous pathogen, toxin and etc, and quickly respond to re-educate immune cells and transfer attack signal to other organs, when this process is overreacted, liver itself and the connected organs can suffer injury, which aggravates sepsis at the beginning phase. This process can be reversible as liver has strong regeneration and compensatory ability to adapt to septic stress in most conditions, thus may probably not affect mortality of sepsis. Early cardiovascular and respiration dysfunction are independent risk factor for septic patients after discharge (Table 4) possibly because cardiovascular and respiratory organs are short of compensatory ability as liver.

Our research has substantial strengths. To our best knowledge, this is the first report that early liver injury is closely related to organ failure of sepsis in the world from clinical data. Second, we adopt transaminase as indicator for early hepatic injury which has high sensitivity to screening out latent liver injury cases in early stage of sepsis, we suppose this is objective and scientific. Third, we find younger people are prone to have early liver injury compared to older ones in sepsis in Chinese Han population which is different from other racial results.

There are several limitations to our study. First, the study design is retrospective and the sample size is relative small, so bias can not be eliminated. We have tried our best to strengthen data integrity and guarantee date equarlity by decreasing loss to follow-up and use multivariate regression analysis and multivariate Cox hazard analysis to reduce bias as much as possible. Secondly, the increasing sensisivity by using transaminase for detecting early hepatic injury also reduce specifity to recognize real liver injury thus brings bias. We think it's double-edged sword which is unvoidable for losing perfection at present condition. We emphasis on highlighting the latent and early liver injury state of sepsis which is often overlooked in previous studies. Third, the single centered design limited the generalisability of our conclusion, while we carried out this study in different ICUs setting in our hospital to partially decrease generalisability reduction. Fourth, we have missing data but very few as we have tried our best to manage and control quarlity of all data from designing phase of this study. 


\section{Conclusion}

Early liver injury within 48 hours on admission is closely related to organ failure in sepsis, but not mortality after discharge. Age, hypertriglyceridemia, serum total bile acid, hemoglobin and renal dysfunction were the independent predictors for early liver injury in sepsis. And Age, organ failure of cardiovascular dysfunction, respiration dysfunction were independent risk factors for mortality of septic patients after discharge. We hope our research will inspire the desire to study the exact work rules of liver intersecting with other organs in the initiation and progress of sepsis. Future clinical research with big data and in-depth basic research are suggested to verify our result and further clarify the underlying mechanism of the effect of early liver injury on sepsis pathogenesis in the future. And whether targeting early liver function can be early preventive or therapeutic strategies for ameliorating organ failure and improve clinical outcomes of sepsis.

\section{Abbreviations}

ICU, intensive care units; SOFA, sequential organ failure assessment; APACHE II, Acute Physiology, Age, Chronic Health Evaluation II; AKI, acute kidney injury; RR, respiratory rate; CRRT, continuous renal replacement therapy; ALT, Alanine transaminase; AST, Aspartate transaminase; TB, total bilirubin; DB, direct bilirubin; GGT, gamma-glutamyl transpeptidase; TBA, total bile acid; PCT, procalcitonin; Lac, lactic acid; CK, creatine kinase; CK-MB, creatine kinase-MB; LDH, lactate dehydrogenase; Mb, myoglobin; APTT, activated partial thromboplastin time; PT, prothrombin time; PTA, prothrombin time activity percentage; GCS, Glasgow Coma Scale; MV, time mechanical ventilation time.

\section{Declarations}

\section{Ethics approval and consent to participate}

The study was approved by the Institutional Review Board of Xiangya Hospital Central South University which abides by the guidance of the Declaration of Helsinki. This was a retrospective study, all data from electronic medical record and we adequately protected patients' privacy, so consent requirements were waived as a result. IRB number 2017121159.

\section{Consent for publication}

Not applicable.

\section{Availability of data and materials}

All data generated or analysed during this study are included in this published article.

\section{Competing interests}

The authors declare that they have no competing interests. 


\section{Funding}

Not applicable.

\section{Authors' contributions}

Peng ML contributed to the conception and design of the research; Qi DS, Liu ZY, Zhao SP and Zhang XLcontributed to the acquisition and analysis of the data; Ai YH, Zhang LN, Zhao SP, and Zhang HS contributed to the interpretation of the data; and Peng ML and Qi DS drafted the manuscript. All authors critically revised the manuscript, agree to be fully accountable for ensuring the integrity and accuracy of the work, and read and approved the final manuscript.

\section{Acknowledgements}

There are no acknowledgements.

\section{References}

1. Hotchkiss RS, Monneret G, Payen D. Sepsis-induced immunosuppression: from cellular dysfunctions to immunotherapy. Nature Reviews Immunology. 2013;13(12):862-74.

2. Sakr Y, Jaschinski U, Wittebole X, Szakmany T, Lipman J, Namendys-Silva SA, et al. Sepsis in Intensive Care Unit Patients: Worldwide Data From the Intensive Care over Nations Audit. Open Forum Infect Dis. 2018;5(12):ofy313.

3. Strnad P, Tacke F, Koch A, Trautwein C. Liver - guardian, modifier and target of sepsis. Nat Rev Gastroenterol Hepatol. 2017;14(1):55-66.

4. Yan J, Li S, Li S. The role of the liver in sepsis. Int Rev Immunol. 2014;33(6):498-510.

5. Mayr FB, Yende S, Angus DC. Epidemiology of severe sepsis. Virulence. 2014;5(1):4-11.

6. Ogura H, Gando S, Saitoh D, Takeyama N, Kushimoto S, Fujishima S, et al. Epidemiology of severe sepsis in Japanese intensive care units: a prospective multicenter study. J Infect Chemother. 2014;20(3):157-62.

7. Jalan R, Fernandez J, Wiest R, Schnabl B, Moreau R, Angeli P, et al. Bacterial infections in cirrhosis: a position statement based on the EASL Special Conference 2013. J Hepatol. 2014;60(6):1310-24.

8. Gustot T, Durand F, Lebrec D, Vincent JL, Moreau R. Severe sepsis in cirrhosis. Hepatology. 2009;50(6):2022-33.

9. Gustot T, Felleiter P, Pickkers P, Sakr Y, Rello J, Velissaris D, et al. Impact of infection on the prognosis of critically ill cirrhotic patients: results from a large worldwide study. Liver Int. 2014;34(10):1496503.

10. Singer M, Deutschman CS, Seymour CW, Shankar-Hari M, Annane D, Bauer M, et al. The Third International Consensus Definitions for Sepsis and Septic Shock (Sepsis-3). JAMA. 2016;315(8):80110. 
11. Ferguson ND, Fan E, Camporota L, Antonelli M, Anzueto A, Beale R, et al. The Berlin definition of ARDS: an expanded rationale, justification, and supplementary material. Intensive Care Med. 2012;38(10):1573-82.

12. Kellum JA, Lameire N, Group KAGW. Diagnosis, evaluation, and management of acute kidney injury: a KDIGO summary (Part 1). Crit Care. 2013;17(1):204.

13. Vincent JL, Moreno R, Takala J, Willatts S, De Mendonca A, Bruining H, et al. The SOFA (Sepsisrelated Organ Failure Assessment) score to describe organ dysfunction/failure. On behalf of the Working Group on Sepsis-Related Problems of the European Society of Intensive Care Medicine. Intensive Care Med. 1996;22(7):707-10.

14. Kramer L, Jordan B, Druml W, Bauer P, Metnitz PG, Austrian Epidemiologic Study on Intensive Care ASG. Incidence and prognosis of early hepatic dysfunction in critically ill patients--a prospective multicenter study. Crit Care Med. 2007;35(4):1099-104.

15. Chan HL, Kwan AC, To KF, Lai ST, Chan PK, Leung WK, et al. Clinical significance of hepatic derangement in severe acute respiratory syndrome. World J Gastroenterol. 2005;11(14):2148-53.

16. Ebert EC. Hypoxic liver injury. Mayo Clin Proc. 2006;81(9):1232-6.

17. Starr ME, Saito H. Sepsis in old age: review of human and animal studies. Aging Dis. 2014;5(2):12636.

18. Koskinas J, Gomatos IP, Tiniakos DG, Memos N, Boutsikou M, Garatzioti A, et al. Liver histology in ICU patients dying from sepsis: a clinico-pathological study. World J Gastroenterol. 2008;14(9):138993.

19. Moreno R, Vincent JL, Matos R, Mendonca A, Cantraine F, Thijs L, et al. The use of maximum SOFA score to quantify organ dysfunction/failure in intensive care. Results of a prospective, multicentre study. Intens Care Med. 1999;25(7):686-96.

20. Reith HB, Mittelkotter U, Wagner R, Thiede A. Procalcitonin (PCT) in patients with abdominal sepsis. Intens Care Med. 2000;26:S165-S9.

21. Novotny S, Lee-Plenty N, Wallace K, Kassahun-Yimer W, Jayaram A, Bofill JA, et al. Acute kidney injury associated with preeclampsia or hemolysis, elevated liver enzymes, and low platelets syndrome. Pregnancy Hypertens. 2020;19:94-9.

22. Raj D, Tomar B, Lahiri A, Mulay SR. The gut-liver-kidney axis: Novel regulator of fatty liver associated chronic kidney disease. Pharmacol Res. 2019;152:104617.

23. de Stoppelaar SF, van't Veer C, Claushuis TAM, Albersen BJA, Roelofs JJTH, van der Poll T. Thrombocytopenia impairs host defense in gram-negative pneumonia-derived sepsis in mice. Blood. 2014;124(25):3781-90.

24. Broadley SP, Plaumann A, Coletti R, Lehmann C, Wanisch A, Seidlmeier A, et al. Dual-Track Clearance of Circulating Bacteria Balances Rapid Restoration of Blood Sterility with Induction of Adaptive Immunity. Cell Host Microbe. 2016;20(1):36-48.

25. Claushuis TAM, van Vught LA, Scicluna BP, Wiewel MA, Klouwenberg PMCK, Hoogendijk AJ, et al. Thrombocytopenia is associated with a dysregulated host response in critically ill sepsis patients. 
Blood. 2016;127(24):3062-72.

26. Yu C, Xu C, Xu L, Yu J, Miao M, Li Y. Serum proteomic analysis revealed diagnostic value of hemoglobin for nonalcoholic fatty liver disease. J Hepatol. 2012;56(1):241-7.

27. Chung KW, Kim KM, Choi YJ, An HJ, Lee B, Kim DH, et al. The critical role played by endotoxininduced liver autophagy in the maintenance of lipid metabolism during sepsis. Autophagy. 2017;13(7):1113-29.

28. Jenne CN, Kubes P. Immune surveillance by the liver. Nat Immunol. 2013;14(10):996-1006.

29. Lendemans S, Heuer M, Nast-Kolb D, Kuhne CA, Dammann M, Lefering R, et al. [Significance of liver trauma for the incidence of sepsis, multiple organ failure and lethality of severely injured patients. An organ-specific evaluation of 24,771 patients from the trauma register of the DGU]. Unfallchirurg. 2008;111(4):232-9.

\section{Figures}

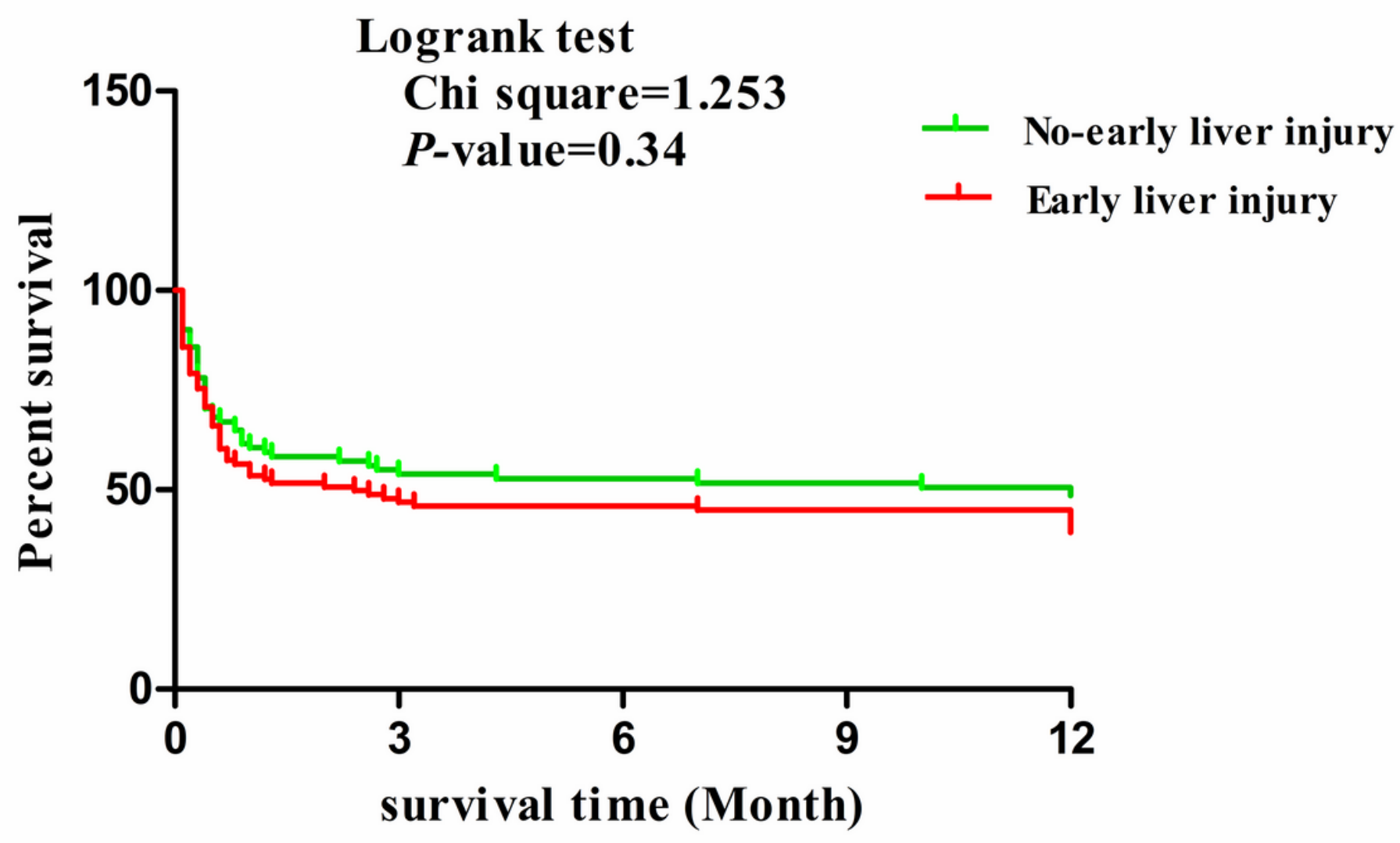

Figure 1

Time to death analysis. The figure shows the survival curves for all included patients at 1 year after randomization of the last patients. Kaplan-Meier analysis showed survival time did not differ between the early liver injury group and the normal group. Log rank $P=0.34$. 
Page 20/20 